\title{
UFC-Inventor: Uma Ferramenta de Autoria Dirigida por Modelos para Geração de Aplicações Ubíquas para Aulas de Campo
}

\author{
UFC-Inventor: A Model Driven Authoring Tool to Generation of Ubiquitous Applications for \\ Field Trips
}

\author{
Edgar Marçal \\ Instituto Universidade Virtual \\ Universidade Federal do Ceará \\ edgar@virtual.ufc.br
}

\author{
Rossana M. de Castro Andrade \\ Departamento de Computação \\ Universidade Federal do Ceará \\ rossana@ufc.br
}

\author{
Windson Viana \\ Instituto Universidade Virtual \\ Universidade Federal do Ceará \\ windson@virtual.ufc.br
}

\section{Resumo}

As aulas de campo promovem as habilidades dos estudantes ao incrementarem a capacidade de observação $e$ descoberta em várias áreas do conhecimento. Ao inserir tecnologias ubíquas nas aulas de campo, como sensores e atuadores, estas aulas apresentam benefícios quando comparadas às aulas de campo tradicionais. Entretanto, no contexto destas aulas, o desenvolvimento de sistemas ubíquos impõe desafios como o tratamento da heterogeneidade dos dispositivos móveis, a ausência de infraestrutura de comunicação fixa, o ambiente computacional dinâmico e o suporte à mobilidade do usuário. Este artigo apresenta o UFC-Inventor, uma ferramenta voltada para professores utilizarem os recursos dos dispositivos móveis e da Computação Ubíqua (como câmera, GPS, bússola e acelerômetro) em aulas de campo. O UFC-Inventor possibilita a geração automática de aplicações multiplataforma sem a necessidade de o usuário ter conhecimentos de programação. Além do desenvolvimento da ferramenta de autoria, outra contribuição importante desse trabalho foi a realização de testes de usabilidade com os públicos-alvo da abordagem. Primeiramente com os professores, observaram-se bons níveis de usabilidade e o interesse em usar o UFC-Inventor em suas aulas frequentemente. Posteriormente, uma aplicação ubíqua gerada pelo UFC-Inventor foi testada pelos estudantes em uma aula de campo de Geologia, sendo aprovada nos critérios de usabilidade e utilidade.

Palavras-Chave: Desenvolvimento dirigido por modelos, Computação Ubíqua, mobile learning, aula de campo.

\begin{abstract}
Field trips promote student skills by increasing the ability of observation and discovery in various areas of knowledge. By incorporating ubiquitous technologies, such as sensors and actuators, in field trips, they show benefits when compared to traditional classes. Ubiquitous applications for field trips imply to deal with device heterogeneity, absence of fixed communication infrastructure, dynamic computational environment, and support for user mobility. This paper presents UFC-Inventor, a tool for teachers to be able to use the resources of mobile devices and ubiquitous computing (such as camera, GPS, compass, and accelerometer) in their field trips. UFC-Inventor enables the automatic generation of cross-platform applications without the need of the user to have specific knowledge about programming. In addition to the development of the authoring tool, another significant contribution of this work was the performance of usability tests with distinct audiences. Teachers have indicated proper levels of usability, highlighting the interest in using UFC-Inventor in their classes frequently. Besides, we tested a ubiquitous application generated by UFC-Inventor with the students in a Geology field trip. They approved the application concerning its usability and utility.
\end{abstract}

Keywords: Model driven development, ubiquitous computing, mobile learning, field trips.

Cite as: Marçal, E., Andrade, R. M. de C. \& Viana, W. (2019). UFC-Inventor: A Model Driven Authoring Tool to Generation of Ubiquitous Applications for Field Trips (UFC-Inventor: Uma Ferramenta de Autoria Dirigida por Modelos para Geração de Aplicações Ubíquas para Aulas de Campo). Brazilian Journal of Computers in Education (Revista Brasileira de Informática na Educação - RBIE), 27(3), 132-149. DOI: 10.5753/RBIE.2019.27.03.132 


\section{Introdução}

A aula de campo proporciona diversos benefícios à aprendizagem, caracterizando-se como uma ação motivadora que permite aos alunos vivenciarem na prática o que foi lecionado em sala de aula (Shakil \& Hafeez, 2011). Ela favorece uma abordagem ao mesmo tempo mais complexa e menos abstrata dos fenômenos estudados em diferentes domínios do conhecimento, ampliando a capacidade de observação e descoberta dos alunos. As atividades desenvolvidas durante as aulas de campo são essenciais para a aprendizagem em diversos cursos de graduação (e.g. Geologia, Geografia e Engenharia de Minas) e também são importantes práticas educativas no ensino básico, em assuntos como ecologia e meio ambiente.

Durante as aulas de campo, alunos e professores se deparam com dificuldades que podem comprometer os benefícios dessas práticas educativas (Roslin et al., 2009; Shakil \& Hafeez, 2011). Em determinadas situações, os alunos ficam sobrecarregados com o excesso de atividades (e.g. analisar e registrar as informações do ambiente, anotar as observações do professor e realizar as atividades em grupo), além de terem de carregar diversos equipamentos (e.g. GPS, bússola, câmera digital e caderneta de campo). Além disso, os professores, muitas vezes, têm de lidar com turmas numerosas e alunos dispersos no campo, o que pode resultar em um suporte inadequado à aprendizagem dos estudantes.

Uma alternativa para contornar os obstáculos encontrados nas aulas de campo tem sido o uso das tecnologias da Computação Ubíqua (Gilman et al, 2015; Marçal et al., 2014; Wu, Hwang \& Tsai, 2013; Viana et al., 2011). Em seu artigo seminal (Weiser, 1999), Mark Weiser introduziu o termo Computação Ubíqua para preconizar que em futuro próximo os sistemas computacionais estariam "embutidos” em elementos da vida diária, sendo capazes de trocar dados entre si com a menor intervenção possível dos usuários. Em sua visão de computação, o usuário interage com interfaces de forma natural e sem se dar conta da presença de computadores, sensores e atuadores espalhados pelo ambiente, estando a tecnologia completamente inserida no seu dia a dia de forma calma e invisível.

A inserção desses recursos nas atividades em campo pode melhorar os processos de ensino e aprendizagem nas aulas, destacando-se: a identificação do contexto do aluno para entrega de conteúdos e exercícios de acordo com a situação dele naquele momento; o registro das ações e preferências dos aprendizes para recomendações futuras em atividades similares; a utilização de sensores para coleta e anotação de informações relevantes à aprendizagem em campo; e, a comunicação entre alunos e professores por meio de redes sem fio ad hocs.

Entretanto, o desenvolvimento de sistemas ubíquos não é uma tarefa trivial que pode ser realizada por pessoas sem conhecimentos específicos de programação (Rocha et al., 2011). Os requisitos particulares desses sistemas impõem desafios para a sua implementação, tais como: tratamento de forma transparente da heterogeneidade dos dispositivos; intermitência de infraestrutura de comunicação; dinamicidade dos elementos computacionais do ambiente e o suporte à mobilidade dos usuários (Gilman et al, 2015; Hwang \& Wong, 2014). Além disso, cada aula de campo varia de acordo com o local, com os participantes e com o que o docente pretende explorar naquele ambiente (de Oliveira \& Correia, 2013). Assim, para que as tecnologias ubíquas possam ser usadas em campo, existe a necessidade de soluções adaptadas a contextos de aprendizagem específicos. Duas abordagens utilizadas para o desenvolvimento de sistemas ubíquos que têm entre seus benefícios a abstração dos aspectos complexos da programação são: a Engenharia Dirigida por Modelos (em inglês, Model-Driven Engineering - MDE) (Brambilla, Cabot \& Wimmer, 2012) e as ferramentas de autoria (Rachid \& Ishitani, 2012). 
Esse artigo apresenta o UFC-Inventor (Ubiquitous Field Classes - Inventor), um sistema voltado para professores (sem conhecimentos de programação) criarem, gerarem e disponibilizarem aplicações ubíquas para serem utilizadas pelos seus alunos em aulas de campo. O sistema foi avaliado com o objetivo de se responder as seguintes questões de pesquisa: QP1 Professores conseguiriam utilizar o UFC-Inventor para modelar e gerar aplicações com recursos de computação ubíqua para serem usadas em aulas de campo? QP2 - Um professor, com o auxílio do UFC-Inventor, seria capaz de gerar uma aplicação ubíqua para ser usada por alunos em uma aula de campo real e que fosse fácil de usar e útil para os discentes?

\section{Trabalhos Relacionados}

As tecnologias da Computação Ubíqua têm sido utilizadas de forma efetiva para favorecer as aulas de campo e as práticas educativas em geral. Entretanto, o desenvolvimento de aplicações com esses recursos não é uma tarefa trivial e existem algumas soluções que se propõem a facilitar essa implementação, tais como: Lemonade (Giemza, Bollen \& Hoppe, 2011), UoLmP (Gómez, 2013), MAT for ARLearn (Tabuenca et al., 2016), Midgar (García et al., 2014) e App Inventor (Xie \& Abelson, 2016). A Tabela 1 mostra um comparativo entre o UFC-Inventor e essas ferramentas, utilizando-se como critérios características importantes para ferramentas de autoria de aplicações educativas ubíquas (Tabuenca et al., 2016).

Tabela 1. Comparação entre o UFC-Inventor e ferramentas de autoria similares.

\begin{tabular}{|c|c|c|c|c|c|c|}
\hline $\begin{array}{l}\text { Ferramentas } \\
\text { Critérios }\end{array}$ & LEMONADE & UoLmP & $\begin{array}{l}\text { MAT for } \\
\text { ARLearn }\end{array}$ & Midgar & $\begin{array}{c}\text { App } \\
\text { Inventor }\end{array}$ & UFC-Inventor \\
\hline Modelagem gráfica & Sim & Não & Sim & Sim & Sim & Sim \\
\hline $\begin{array}{l}\text { Conhecimentos de } \\
\text { programação }\end{array}$ & Não & Sim & Não & Não & Sim & Não \\
\hline $\begin{array}{l}\text { Utilização de } \\
\text { Especificações }\end{array}$ & Não & IMS LD & Não & Não & Não & ML4UL/ IMS LD \\
\hline Modelo aberto & Não & Sim & Não & Não & Não & Sim \\
\hline Compartilhamento & Não & Não & Sim & Sim & Sim & Sim \\
\hline $\begin{array}{l}\text { Sistema operacional } \\
\text { móvel }\end{array}$ & Windows & Android & Android & Android & Android & $\begin{array}{l}\text { Android, Windows } \\
\text { Phone, iOS, entre } \\
\text { outros. }\end{array}$ \\
\hline Uso de sensores & GPS & * & GPS & $* *$ & $\begin{array}{c}\text { GPS, NFC, } \\
\text { Bússola e } \\
\text { Acelerômetro }\end{array}$ & $\begin{array}{l}\text { GPS, QR Code, } \\
\text { Bússola, *** }\end{array}$ \\
\hline $\begin{array}{l}\text { Informação } \\
\text { Contextual }\end{array}$ & Localização & * & Localização & $* *$ & $\begin{array}{l}\text { Localização e } \\
\text { Orientação }\end{array}$ & $\begin{array}{l}\text { Localização, Código } \\
\text { QR, Orientação, *** }\end{array}$ \\
\hline $\begin{array}{l}\text { Dependência de } \\
\text { conexão }\end{array}$ & Não & Sim & Sim & Sim & Não & Não \\
\hline Interação sem fio & Não & Não & Não & Não & Sim & Sim \\
\hline
\end{tabular}

* Como a abordagem proposta no trabalho UoLmP (Gómez, 2013) não prevê uma ferramenta de edição, não é possível a inclusão de sensores direta no projeto.

** Tipos de sensores e informações contextuais dependem dos objetos inteligentes existentes no servidor (García et al., 2014).

*** A linguagem ML4UL e o ambiente UFC-Inventor podem ser estendidos para suportar outros sensores e informações contextuais. Além disso, por meio das perguntas, que são suportadas tanto pela linguagem quanto pelo ambiente, é possível criar sensores virtuais que podem ser usados na aplicação para obter informações contextuais como localização e hora do dia. 
É importante destacar que, comparado aos trabalhos relacionados, o UFC-Inventor é o único capaz de gerar aplicações com recursos de Computação Ubíqua para distintos sistemas operacionais com uso de uma modelagem gráfica. Ele também não demanda conhecimentos de programação.

Vale ressaltar outro diferencial importante do UFC-Inventor: a utilização de uma especificação aberta que possibilita a interoperabilidade com outras ferramentas. Padrões abertos (ou especificações abertas) são aqueles que estão publicamente disponíveis e não são controlados por nenhuma corporação ou governo, permitindo que empresas, pessoas e países possam utilizá-los com autonomia (Chede, 2008). Outra qualidade importante do UFC-Inventor é o fato das aplicações geradas não dependerem de Internet para funcionar. Essa característica é particularmente importante em aulas de campo, uma vez que muitas vezes acontecem longe dos centros urbanos e sem conexão com a Internet.

\section{Metodologia}

\subsection{Concepção da Ferramenta}

Esta pesquisa pode ser caracterizada como estudo exploratório que visa investigar o potencial da aplicação de sistemas ubíquos em aulas de campo de Geociências. A metodologia seguida neste trabalho se baseou, principalmente, no processo de Design Centrado no Usuário. Nesta metodologia, o desenvolvedor não só imagina como será a interação do usuário final com o sistema em construção como também realiza uma série de testes com usuários reais para dar validade ao trabalho ao longo do processo de desenvolvimento (Garret, 2011).

Esta pesquisa decorre, portanto, de estudos contínuos realizados junto ao Departamento de Geologia da Universidade Federal do Ceará desde 2012. Primeiramente, buscou-se introduzir tecnologias móveis nas aulas de campo usando aplicações já existentes. Após várias reuniões e sessões de Brainstorming, aplicou-se tablets em uma aula de campo. Em seguida, a partir dos problemas encontrados, foi desenvolvido e testado um aplicativo, o Geomóvel (Marçal et al., 2014), para auxiliar as etapas de anotação das aulas de campo. As lições aprendidas nestas fases culminaram na criação de uma ferramenta para auxiliar o próprio professor a criar aplicativos móveis semelhantes ao Geomóvel, sendo esta ferramenta e seu processo de avaliação descritos neste artigo.

\subsection{Teoria da aprendizagem auxiliada por Computação Móvel}

Pesquisas sobre aprendizagem com suporte a tecnologias móveis já existiam mesmo antes da criação do PC. Atualmente, as aplicações de m-learning podem ser vistas como onipresentes devido ao uso contínuo dos smartphones. Ou seja, de certa forma, elas já incorporaram conceitos preconizados por Mark Weiser. Além disso, métodos de Computação Ubíqua podem identificar o contexto do aluno e sugerir conteúdo, atividades e dicas para melhorar o aprendizado de acordo com o que foi capturado, ampliando assim o seu potencial de uso. Ao considerar as aulas de ciências que acontecem além das paredes da sala de aula, a Computação Ubíqua se apresenta como uma tecnologia ainda mais promissora por permitir que os alunos acessem materiais no campo (onipresença), no local e momentos apropriados (e.g. um vídeo que deve ser exibido ao entardecer) e de acordo com contexto em que se encontram (e.g. em frente a uma rocha vulcânica cuja composição o aluno ainda não documentou).

Desta forma, a pesquisa aqui apresentada pode ser vista como uma evolução, natural, das pesquisas de m-learning. Ela se pauta na Teoria da Aprendizagem Móvel proposta por Sharples 
et al. (2006), que preconiza que a educação entrou na terceira onda tecnológica com dispositivos móveis amigáveis, onipresentes, fáceis de usar, pessoais e transportáveis a todo lugar. A teoria de Sharples et al. (2006) segue princípios na Teoria Conversacional. Neste modelo conversacional, estão integrados quatro processos: o debate, a interação, a reflexão e a adaptação. A conversação e o contexto tornam-se conceitos essenciais desses processos e são ainda mais potencializadas nas aulas de campo, que por definição quebram o modelo de ambiente controlado das salas de aula. Além disso, essa teoria é inspirada também em elementos da teoria da aprendizagem de Vygotsky (1978). De acordo com Vygotsky, o conhecimento se faz pela mediação por meio de sujeitos e de objetos do mundo que rodeiam o aluno. Assim, as tecnologias de m-learning ampliam esta percepção e também podem amplificar o papel mediador do professor. Neste ponto, em especial, é que o UFC-Inventor se apresenta, visto que seu foco é potencializar o papel do professor dandolhe uma ferramenta para gerar suas próprias aplicações móveis de campo.

\subsection{Avaliação da Ferramenta}

Dois tipos de avaliação são reportados neste artigo. Uma primeira avaliação visando obter a percepção dos professores sobre o UFC-Inventor. Cinco professores avaliariam a usabilidade da ferramenta e analisaram o seu potencial de uso em campo. Uma segunda avaliação consistiu em um estudo de caso que avaliou tanto o processo de geração como o uso da aplicação gerada em campo. Esta avaliação ocorreu em um curso de graduação em Geologia da UFC, contando com a participação do professor da disciplina e de dezessete alunos na aula de campo.

\section{A Ferramenta}

O UFC-Inventor consiste em um sistema composto por três componentes principais: i) o módulo UFC-GLM (Graphical Learning Modeller), para modelagem gráfica das aulas de campo com recursos da computação ubíqua; ii) o módulo UFC-Generator, para geração e disponibilização das aplicações ubíquas; iii) a linguagem específica de domínio ML4UL (Marçal, Andrade \& Viana, 2015), para integração entre os dois módulos e interoperabilidade com outras ferramentas. Um vídeo com uma demonstração de uso do UFC-Inventor, desde a modelagem até a geração, é apresentado no link: https://vimeo.com/240189660.

A Figura 1 mostra uma visão geral do UFC-Inventor. O objetivo central da ferramenta é proporcionar que professores possam enriquecer suas aulas de campo através de aplicações que utilizem os recursos dos dispositivos móveis dos alunos e da computação ubíqua. O módulo UFCGLM consiste em uma ferramenta de modelagem na qual o projetista (e.g. professor ou especialista de área) pode realizar as seguintes ações: modelar, por meio do editor gráfico (1), as atividades de aprendizagem e o fluxo que elas devem acontecer; inserir na especificação recursos de Computação Ubíqua, como dados provenientes de sensores e comunicação sem fio (2); e, converter (3) o modelo gráfico elaborado com os recursos adicionados para uma especificação no formato ML4UL (4). 


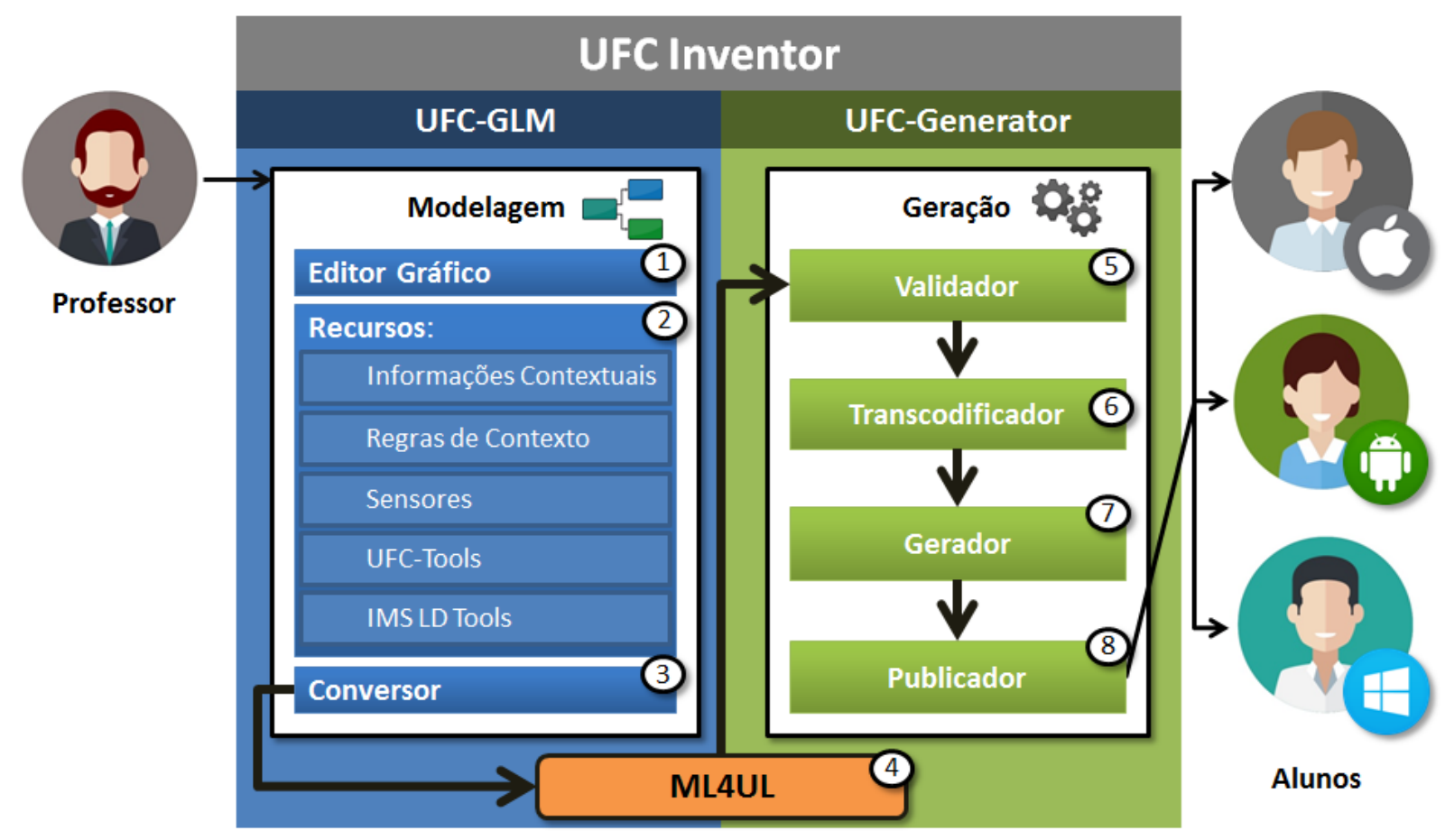

Figura 1. Visão geral do UFC-Inventor.

A utilização da abordagem de desenvolvimento dirigido por modelos (MDE) traz benefícios importantes para o ambiente UFC-Inventor. Primeiramente, o modelo gráfico possibilita uma representação em alto nível da aula de campo ubíqua com elementos que estão mais próximos dos conceitos do domínio (e.g. atividades e fluxo de aprendizagem). Dessa forma, não se exige, de quem está criando a aula, conhecimentos sobre linguagens de programação para gerar as aplicações ubíquas.

Além disso, o uso da linguagem específica de domínio ML4UL, como um modelo intermediário entre o diagrama da aula e o código-fonte, proporciona uma diminuição da dependência da tecnologia. Com isso, por exemplo, no caso da descontinuidade da linguagem de programação utilizada (HTML5 e Javascript), para que o UFC-Inventor continue funcionando basta alterar a função de transcodificação. Nessa situação, o Transcodificador deverá transformar a especificação para uma outra linguagem e em seguida chamar o compilador.

Outra vantagem do UFC-Inventor é o fato de sua estrutura, baseada em dois softwares distintos, facilitar a interoperabilidade com outras ferramentas. Assim, por exemplo, um editor gráfico de terceiros pode ser utilizado para modelar a aula de campo ubíqua, gerar a especificação no formato ML4UL e executar o módulo UFC-Generator para gerar as aplicações ubíquas. De outra forma, pode-se usar o módulo UFC-GLM para especificar uma aula de campo e, depois, a codificação em ML4UL servir com entrada para outra ferramenta de geração de aplicações ou um software interpretador no próprio dispositivo móvel.

A Figura 2 mostra as transformações entre os modelos que acontecem no ambiente UFCInventor, separadas pelos módulos. Parte-se de uma especificação de mais alto nível e independentemente de plataforma. Esse modelo gráfico é convertido para uma codificação textual em ML4UL. Posteriormente, essa especificação é transformada em um modelo baseado em código-fonte de uma linguagem de programação (HTML5 e Javascript). Finalmente, a última transformação é realizada e obtém-se o arquivo com a codificação binária da aplicação móvel. 


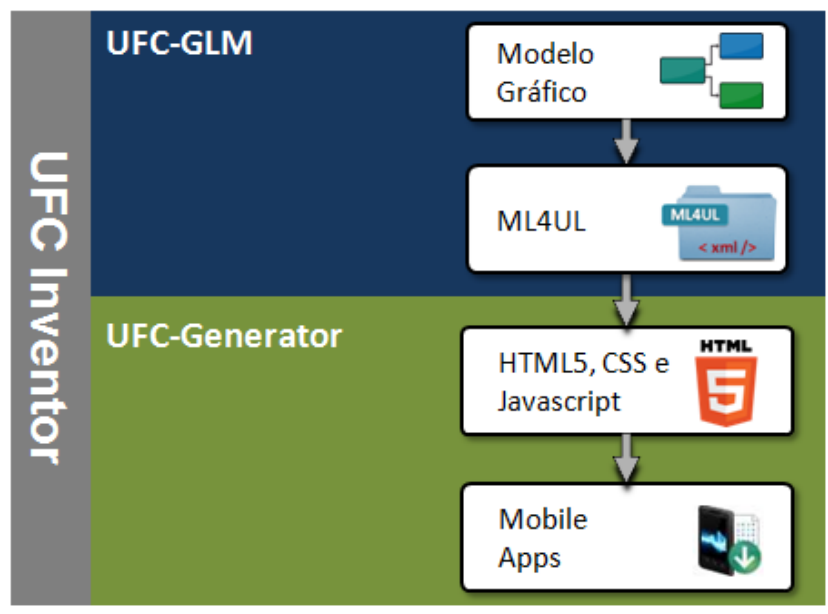

Figura 2. Transformações entre modelos no UFC-Inventor.

O fato de o UFC-Inventor gerar também o código-fonte e não apenas o arquivo binário do aplicativo móvel é outra característica relevante do ambiente. Com isso, o código gerado pode ser reutilizado e alterado, para se produzir outras aplicações com requisitos diferentes dos projetados inicialmente. Com relação a isso, é importante destacar dois aspectos: ela é uma funcionalidade voltada para programadores que detém conhecimentos sobre as linguagens nas quais as aplicações são geradas; e, como esse código gerado precisa ser alterado com uma ferramenta de programação externa ao UFC-Inventor, essas alterações não serão refletidas no modelo original.

\subsection{O Módulo UFC-GLM}

A Figura 3 mostra a tela principal do UFC-GLM. Pode-se observar a paleta com as opções de conexão para o modelo da aula (lado direito) e o ambiente de autoria com um exemplo de diagrama que ilustra o uso de alguns recursos multimídia. Também se pode visualizar na figura a opção “Templates” (lado direito inferior), que contém um modelo pronto para ser reutilizado. Na parte inferior da imagem, são exibidos os tipos de usuários permitidos na aula que está sendo planejada (nesse exemplo, professor e aluno). A Tabela 2 mostra os recursos disponíveis no módulo UFC-GLM.

Tabela 2. Recursos disponibilizados pelo UFC-GLM para as aplicações ubíquas.

\begin{tabular}{ll}
\hline Recurso & Funcionalidade \\
\hline Captura multimídia & $\begin{array}{l}\text { Especificar os mecanismos de captura no dispositivo móvel que serão usados pela } \\
\text { aplicação, como fotos, vídeos e áudio. }\end{array}$ \\
\hline Conteúdo digital & $\begin{array}{l}\text { Descrever quais os recursos multimídia que serão disponibilizados pela aplicação, tais } \\
\text { como imagens, vídeos e áudios. }\end{array}$ \\
\hline GPS & $\begin{array}{l}\text { Permitir que a aplicação utilize o sensor GPS do dispositivo móvel para capturar os } \\
\text { dados de localização geográfica do usuário. }\end{array}$ \\
\hline Bússola & $\begin{array}{l}\text { Possibilitar que a aplicação utilize o sensor de campo magnético do dispositivo para } \\
\text { simular uma bússola e fornecer informações para orientação do aluno. }\end{array}$ \\
\hline QR Code & $\begin{array}{l}\text { Equipar a aplicação com o recurso para leitura de códigos QR (Quick Response) por } \\
\text { meio da utilização da câmera do dispositivo móvel e fornecer informações ao aluno. }\end{array}$ \\
\hline Compartilhamento & $\begin{array}{l}\text { Possibilitar que os participantes da aula de campo (como alunos e professor) possam } \\
\text { compartilhar arquivos (como fotos e áudios) entre si. }\end{array}$ \\
\hline Conferência local & $\begin{array}{l}\text { Disponibilizar na aplicação o recurso de chat entre os participantes que utilizará as redes } \\
\text { sem fio disponíveis no momento da aula de campo. }\end{array}$ \\
\hline
\end{tabular}




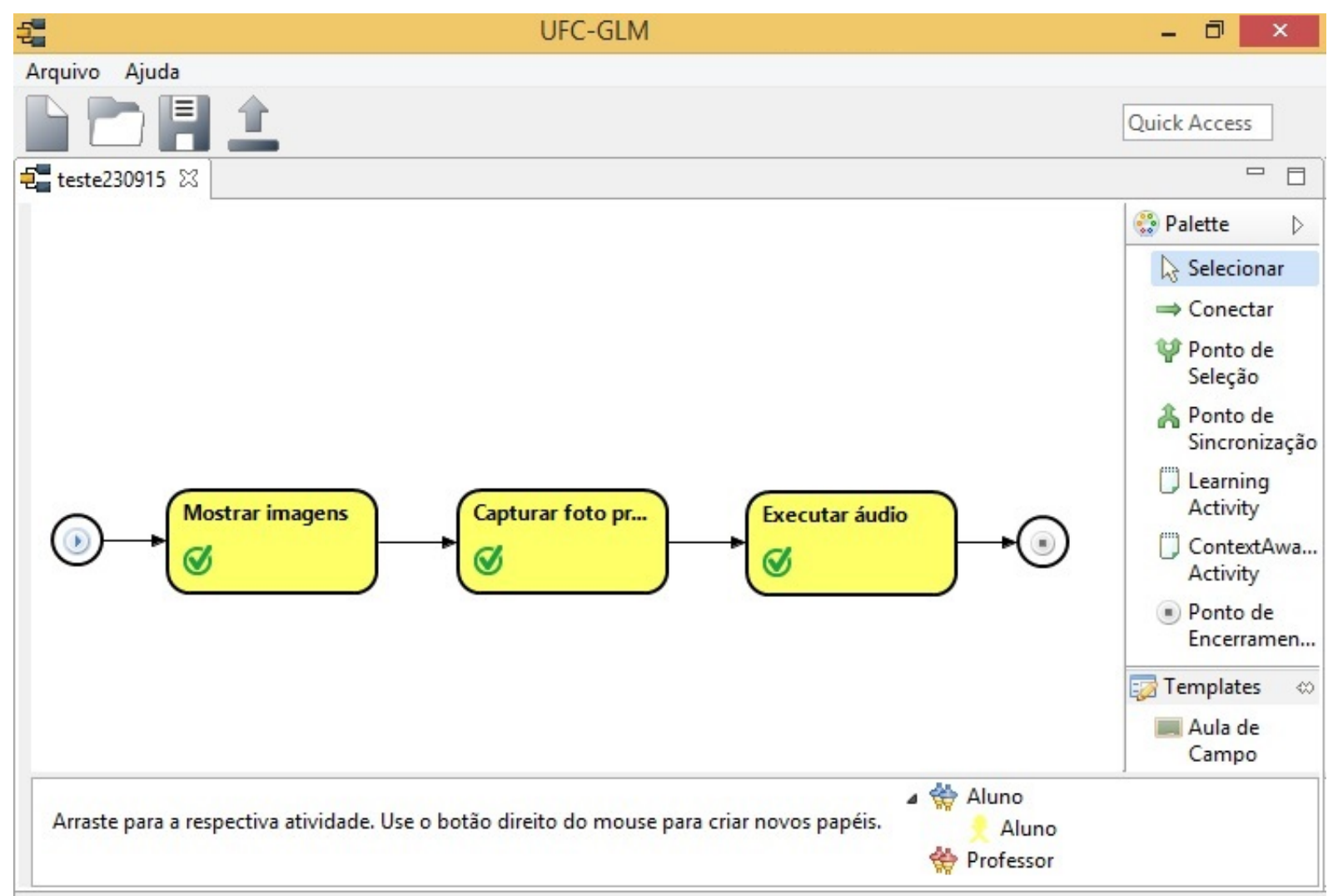

Figura 3. Tela principal do módulo UFC-GLM.

Além disso, o UFC-GLM possibilita a especificação de atividades de aprendizagem condicionadas a regras de adaptação definidas pelo professor em tempo de projeto. Assim, é possível representar uma aula de campo adaptativa e sensível ao contexto dos alunos. A Figura 4 mostra a tela onde o projetista pode configurar as regras de contexto para a aplicação ubíqua. Desta forma, por exemplo, pode-se projetar e gerar uma aplicação que mostre conteúdos específicos (como imagens e animações) ao aluno de acordo com o lugar onde ele se encontra e os locais que ele já visitou. Nesse caso, a variável de controle seria a localização obtida via GPS.

Após concluir a modelagem da aula de campo, com a inclusão de todos os recursos, devese converter o diagrama para o pacote ML4UL. Para isso, o UFC-GLM interpretará o modelo gráfico criado, gerará a especificação ML4UL em XML e a compactará junto com os itens de conteúdo adicionados ao projeto em um arquivo ‘.zip’.

\subsection{A linguagem ML4UL}

A ML4UL (Modeling Language for Ubiquitous Learning) pode ser vista como um metamodelo para representação de aplicações ubíquas. A ML4UL diferencia-se principalmente pelo enfoque dado à representação de atividades de aprendizagem. Para isso, ela se baseia no padrão de especificação educacional IMS Learning Design (IMS LD) (Koper, \& Olivier, 2004). Ao manter a estrutura do IMS LD, a ML4UL herda todas as características dessa especificação. Assim, a representação da aula de campo seguirá um modelo validado no meio acadêmico e adotado em inúmeras instituições. 


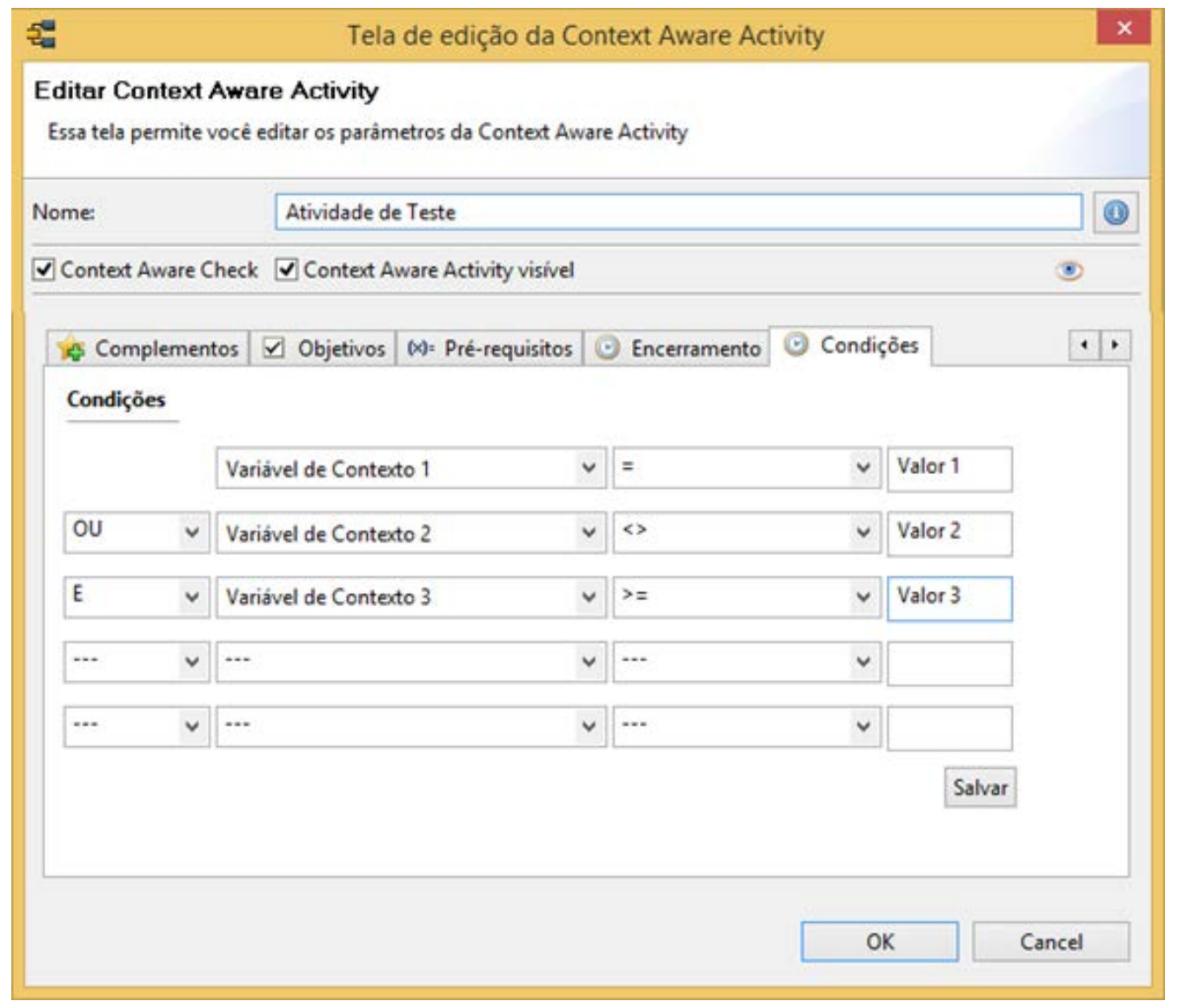

Figura 4. Tela de configuração das regras de contexto no UFC-GLM.

A ML4UL acrescenta ao padrão IMS LD os seguintes elementos voltados especificamente para aplicações ubíquas: <sensor>, <sensor-property>, <contextaware-activity>, <localconference $>, \quad<$ sharing $>, \quad<$ media-capture $>, \quad<$ local-database $>, \quad<$ object-store $>\quad$ e $<$ synchronization>. O projeto de uma aula em ML4UL é composto por: um arquivo em formato XML que contém a especificação da aula de campo (manifesto); e, os arquivos que são necessários para execução das atividades de aprendizagem modeladas, como textos, imagens, áudios e vídeos. Um trecho de código em ML4UL que ilustra o uso do elemento <sensor> é apresentado na Figura 5 .

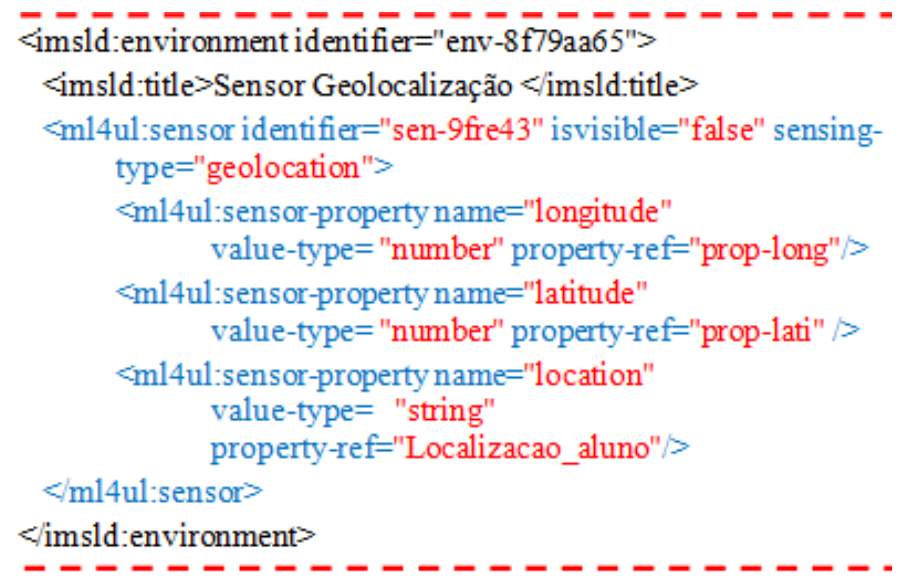

Figura 5. Exemplo de codificação em ML4UL. 


\subsection{O Módulo UFC-Generator}

O módulo UFC-Generator consiste em um sistema Web com uma única tela, mostrada na Figura 6. Nele, o usuário anexa o arquivo com o modelo da aula de campo (gerado no UFC-GLM), informa para qual sistema operacional deseja gerar a aplicação e qual o estilo visual da interface (dentre os disponíveis).

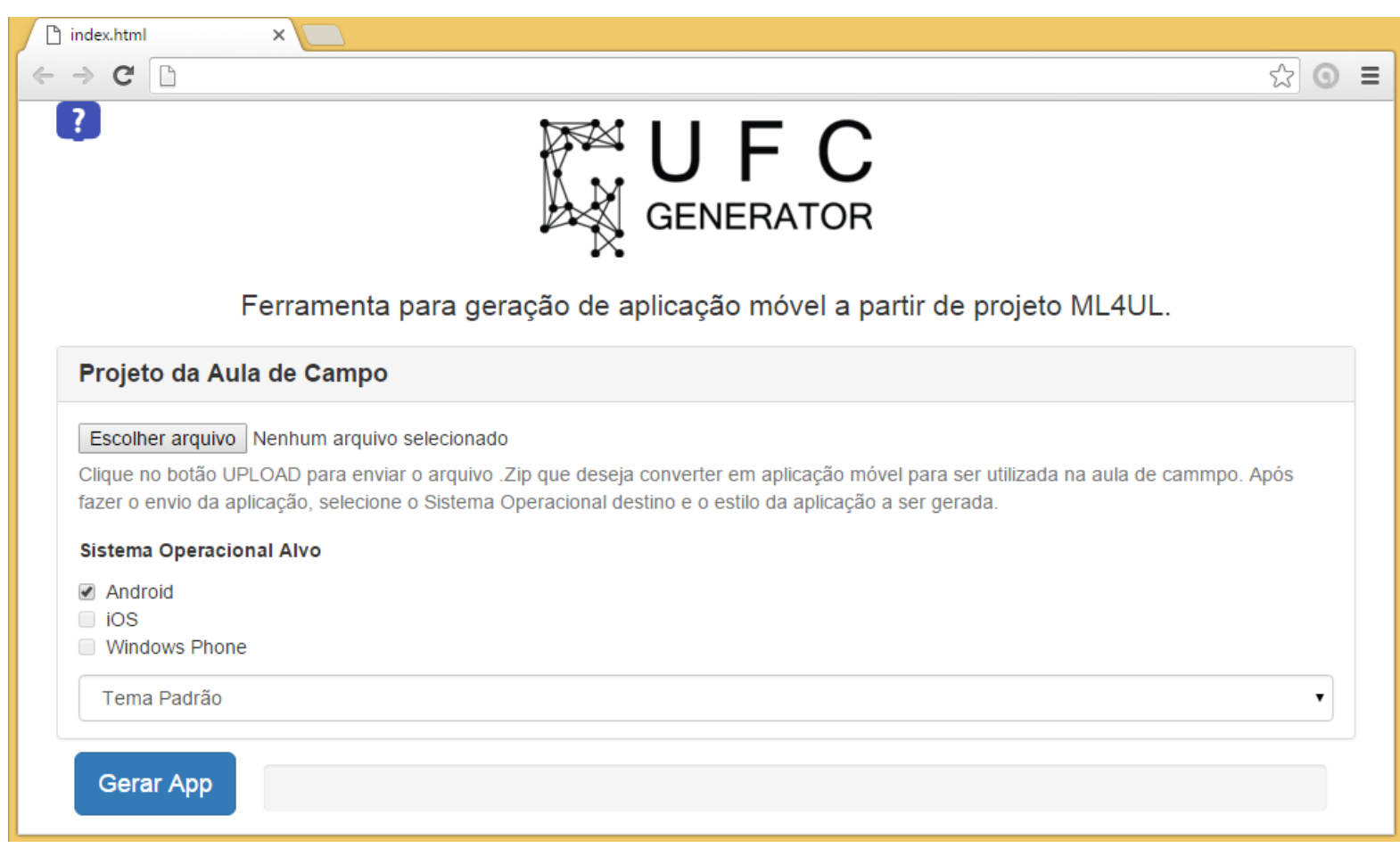

Figura 6. Tela do módulo UFC-Generator.

O arquivo com a codificação da aula de campo ubíqua em ML4UL é a entrada para o módulo UFC-Generator poder gerar as aplicações ubíquas a serem usadas em campo. Para isso, conforme mostrado anteriormente na Figura 1, a especificação deve passar por um módulo validador (5) que faz a leitura do arquivo de entrada e verifica a sua conformidade. Posteriormente, o módulo Transcodificador (6) transforma a especificação da aula de campo de ML4UL para código-fonte em uma linguagem específica. Nessa implementação, a plataforma Apache Cordova $^{\mathrm{TM}}$ foi adotada, e consequentemente, as linguagens HTML5, Javascript e CSS também. Depois, o UFC-Generator executa o Gerador (7), para geração do executável. Por fim, o módulo Publicador (8) identifica o aplicativo gerado e o disponibiliza para os alunos instalarem.

A Figura 7 mostra uma visão geral do processo de transcodificação, que, como se pode observar, é baseado em dois elementos principais: as transformações entre modelos utilizando a linguagem XSLT - eXtensible Stylesheet Language Transformations e a biblioteca de arquivos ML4UL. A partir do arquivo XML com a especificação da aula de campo em ML4UL, são aplicadas transformações que seguem as regras especificadas nos arquivos XSL da biblioteca ML4UL. Como resultados, arquivos HTML e JS (Javascript) são obtidos. Esses especificam o funcionamento da aplicação. Os arquivos Javascript da biblioteca ML4UL contém métodos de acesso às funcionalidades da plataforma Cordova ${ }^{\mathrm{TM}}$ e irão compor o pacote para geração da aplicação final. Os arquivos CSS da biblioteca, por sua vez, constituem os estilos disponíveis que podem ser utilizados para customização da aplicação e são referenciados no arquivo HTML da aplicação. 


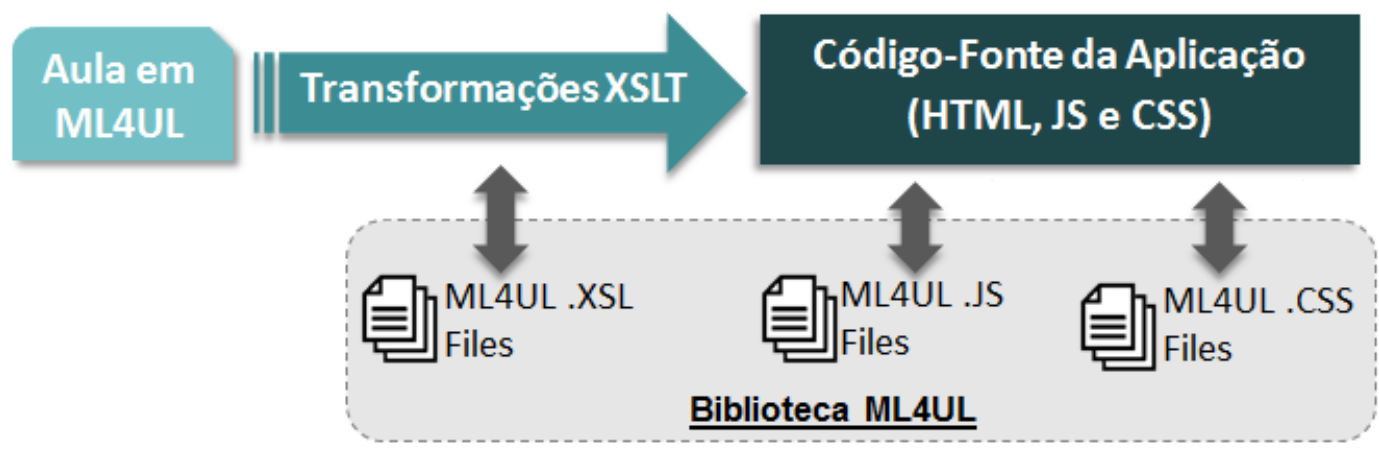

Figura 7. Visão geral do processo de transcodificação.

Após o código ter sido produzido, o UFC-Generator organiza os arquivos fontes, mais os de configuração, executa o Gerador e obtém o executável da aplicação ubíqua (no sistema operacional selecionado). Para disponibilizar as aplicações geradas para os usuários finais, o UFCGenerator apresenta duas opções: um link direto para o arquivo executável da aplicação e o envio de e-mails para os usuários com o arquivo de instalação do aplicativo gerado.

\section{Avaliação}

A análise plena de sistemas de autoria depende da observação dos resultados tanto do software de geração quanto das aplicações produzidas (Ainsworth, S., \& Grimshaw, S. (2004)). Considerando isso, a avaliação do UFC-Inventor foi realizada em duas etapas: verificação da usabilidade do sistema com professores e um estudo de caso em uma aula de campo real com alunos utilizando um aplicativo gerado.

\subsection{Análise de usabilidade do UFC-Inventor}

Para obter a percepção dos professores sobre o UFC-Inventor, foi realizada uma análise específica sobre a usabilidade dos módulos UFC-GLM e UFC-Generator.

\subsubsection{Perfil dos Participantes}

Cinco professores participaram dessa avaliação, com faixa etária entre 35 e 49 anos, que aplicam (ou podem aplicar) aulas de campo em suas disciplinas: um professor do curso de graduação em Geologia da Universidade Federal do Ceará (UFC); um professor do curso de graduação em Zootecnia da UFC; e três professores do ensino fundamental e médio, sendo dois de Geografia e um de Biologia.

\subsubsection{Materiais e Métodos}

Para avaliação da usabilidade do UFC-Inventor, um questionário baseado no System Usability Scale - SUS (Brooke, 1996) foi elaborado. Além de conter os dez itens do SUS, uma questão aberta para os professores relatarem suas opiniões sobre o sistema foi adicionada. As opções de resposta dos dez itens seguiam a escala Likert de cinco pontos, variando de 1 ("Discordo Totalmente”) a 5 (“Concordo Totalmente”). 


\subsubsection{Procedimento}

O processo de avaliação do UFC Inventor ocorreu sequencialmente conforme descrito a seguir. Inicialmente, uma entrevista para identificar o perfil dos participantes foi realizada. Posteriormente, um treinamento para demonstrar o funcionamento e as funcionalidades dos módulos do UFC-Inventor foi conduzido pelo avaliador. Depois, os participantes deveriam realizar três tarefas usando o sistema. Na primeira, cada um deles deveria usar o módulo UFCGLM para modelar uma aula de campo com os recursos de imagem e áudio. A segunda tarefa consistia em usar o módulo UFC-Generator para gerar a aplicação modelada anteriormente. Já na terceira fase, os participantes deveriam realizar o processo completo, modelando uma aula com imagem, áudio e captura de foto usando o UFC-GLM e, depois, gerar a aplicação com o auxílio do UFC-Generator. Por fim, os professores respondiam a um questionário para avaliar o UFCInventor.

\subsubsection{Resultados}

Com relação à avaliação da usabilidade do UFC-Inventor com os professores, a Figura 8 mostra como ocorreu o processo e os tempos de realização das tarefas (o mais rápido e o mais lento). É importante destacar que todos os cinco professores conseguiram realizar (sem auxílio) as três atividades propostas, apesar de apresentarem diferenças nos seus tempos de execução.

A Tabela 3 apresenta os resultados do questionário de usabilidade nas questões baseadas no SUS. As respostas positivas indicam uma boa aceitação do UFC-Inventor pelos professores. Em três questões (1, 7 e 9), todos os participantes responderam positivamente ("Concorda” ou “Concorda Totalmente”) sobre o uso do aplicativo. No geral, a média das respostas foi favorável. As perguntas escritas positivamente tiverem valor de resposta médio igual a 4,16 e as redigidas negativamente tiveram média igual a 1,84.

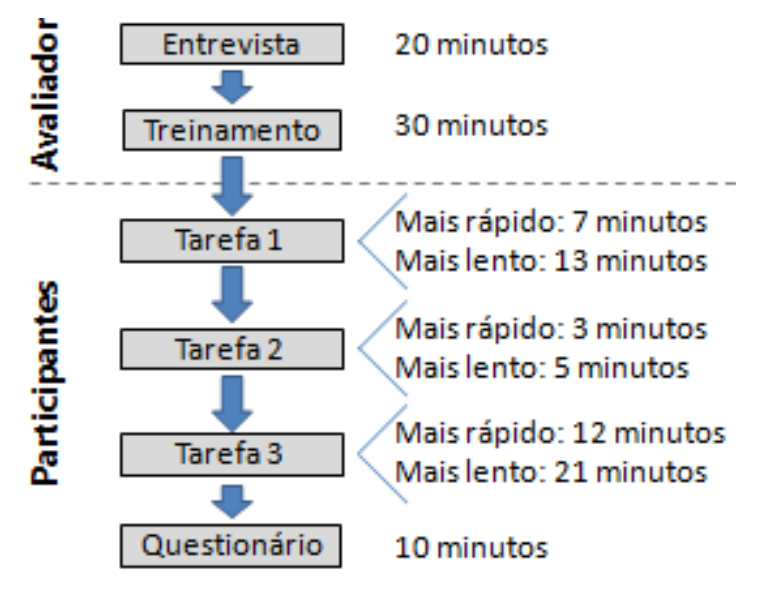

Figura 8. Avaliação da usabilidade do UFC-Inventor com os professores.

A partir da questão aberta presente no instrumento de avaliação, pôde-se obter os principais comentários dos professores sobre a ferramenta. Os seguintes pontos positivos foram indicados: a possibilidade de se criar aplicações educativas com conteúdo multimídia; o fato do sistema não requerer que o usuário tenha conhecimento de programação; e, a capacidade de se gerar aplicações para celulares com distintos Sistemas Operacionais. Dois pontos foram apontados como obstáculos para uso do UFC-Inventor: a impossibilidade de se visualizar como a aplicação está ficando enquanto ela está sendo projetada e a ausência de um manual do usuário detalhado sobre como usar o sistema. 
Tabela 3. Avaliação da usabilidade com os professores.

\begin{tabular}{lcc}
\hline \multicolumn{1}{c}{ Questão } & Média & DP \\
\hline 1) Eu acho que gostaria de usar esse sistema frequentemente. & 4,60 & 0,55 \\
\hline 2) Eu achei esse sistema desnecessariamente complexo. & 1,80 & 0,45 \\
\hline 3) Eu achei o sistema fácil para usar. & 4,00 & 0,71 \\
\hline 4) Eu acho que precisaria do apoio de um suporte técnico para ser possível usar este sistema. & 2,40 & 0,89 \\
\hline 5) Eu achei que as diversas funções neste sistema foram bem integradas. & 3,60 & 0,55 \\
\hline 6) Eu achei que houve muita inconsistência neste sistema. & 2,00 & 0,71 \\
\hline 7) Eu imaginaria que a maioria das pessoas aprenderia a usar esse sistema rapidamente. & 4,40 & 0,55 \\
\hline 8) Eu achei o sistema muito pesado para uso. & 1,20 & 0,45 \\
\hline 9) Eu me senti muito confiante usando esse sistema. & 4,20 & 0,45 \\
\hline 10) Eu precisei aprender uma série de coisas antes que eu pudesse continuar a utilizar esse & 1,80 & 0,45 \\
Sistema. & & \\
\hline
\end{tabular}

\subsection{Estudo de Caso em Campo}

O estudo de caso teve como objetivo principal avaliar o sistema UFC-Inventor considerando o processo completo, desde a modelagem das atividades de aprendizagem, passando pela geração da aplicação, até o uso dela pelos alunos em uma aula de campo real.

\subsubsection{Perfil dos participantes}

Foi escolhida a disciplina “Mapeamento de Terrenos Sedimentares” do curso de graduação em Geologia da UFC. O professor dessa disciplina projetou e gerou uma aplicação multiplataforma para ser usada em campo pelos estudantes, de forma a poder avaliar o funcionamento completo do UFC-Inventor. A turma era composta por um professor e dezessete alunos.

\subsubsection{Procedimento}

Todos os alunos informaram já ter experiência na utilização de aplicativos móveis em smartphones. Cada um deles utilizou a aplicação gerada de forma individualizada, com seus próprios celulares ou outros que foram levados de reserva. Em alguns momentos, os alunos estavam próximos ao professor escutando as orientações. Já em outros, estavam dispersos na região de estudo. O aplicativo gerado era composto por 52 (cinquenta e duas) telas com textos instrutivos, imagens explicativas e perguntas, além das funcionalidades de câmera, bússola, microfone e GPS. Dos dezessete dispositivos utilizados, quinze tinham o sistema operacional Android e dois tinham Windows Phone. A ferramenta também permitia a geração de aplicações para iOS, entretanto essa plataforma não foi utilizada devido ao processo de disponibilização do aplicativo ser mais demorado. Após a aula de campo, os alunos responderam a um questionário com perguntas baseadas no SUS, para verificação da usabilidade e da utilidade da aplicação gerada.

\subsubsection{Resultados}

A partir da realização do estudo de caso em campo pôde-se obter como resultado importante a constatação do funcionamento completo do UFC-Inventor: a aplicação projetada e gerada pelo professor, por meio dos módulos UFC-GLM e UFC-Generator, pôde efetivamente ser utilizada pelos alunos em uma aula de campo real. A Figura 9 ilustra as transformações entre os modelos instanciados para o estudo de caso. São apresentados um diagrama para a aula, uma especificação única na linguagem ML4UL e três diferentes códigos-fonte. Esses códigos tem como base a codificação ML4UL, a partir dos quais foram gerados três executáveis diferentes em duas 
plataformas diferentes: um em Android, no tema 1; outro em Android no tema 2; e outro em Windows Phone no tema 1. Algumas telas da aplicação e o seu funcionamento podem ser visualizados no vídeo: https://vimeo.com/240189660

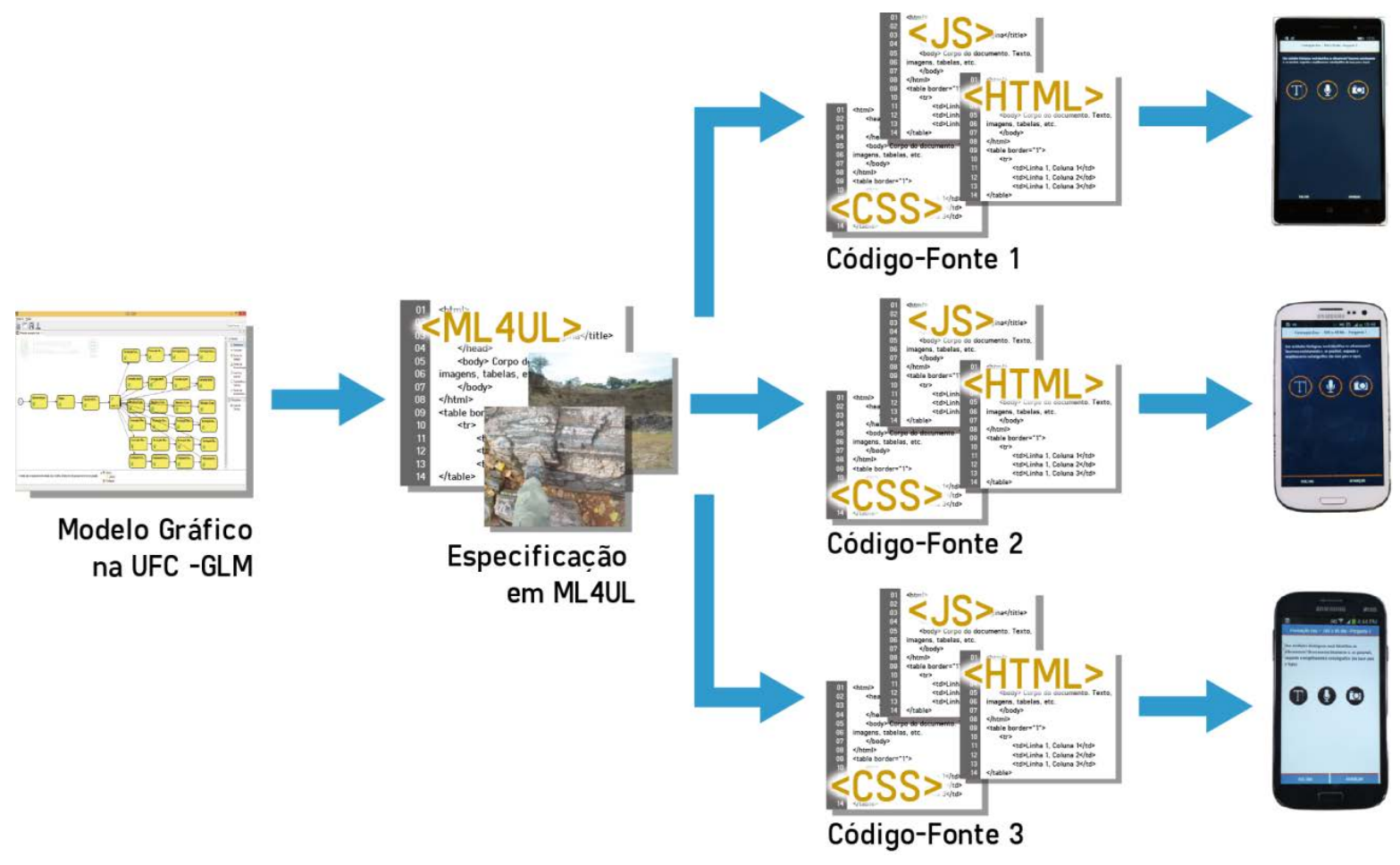

Figura 9. Ilustração do funcionamento do UFC-Inventor através das transformações entre os modelos para o estudo de caso.

Durante o uso da aplicação em campo, os recursos nas três versões tiveram funcionamento semelhante, executando satisfatoriamente. Independentemente do sistema operacional ou do layout utilizado, a navegação entre as telas seguiu a mesma sequência e todas as imagens e textos foram exibidos nos lugares especificados. Todos os dados anotados pelos alunos foram persistidos e os recursos de captura de áudio, de foto e da localização funcionaram da forma como foram projetados, nas versões geradas. Obteve-se um escore SUS médio igual a 83,4, que equivale a um nível excelente de usabilidade (Bangor, Kortum \& Miller, 2009). Além disso, os alunos consideraram que a aplicação foi útil para aquela aula de campo.

\section{Discussão}

As ferramentas de autoria são importantes instrumentos para os educadores transformarem seus conteúdos e disponibilizarem seus materiais, de forma mais fácil e produtiva, nas plataformas digitais atuais (Singh, 2018). Entre os principais benefícios das ferramentas de autoria destacamse: a facilidade de uso, por não requererem do usuário conhecimentos de programação; a economia de tempo, proporcionada pelo ambiente de criação voltado à geração rápida de material didático; e, o acesso em qualquer lugar e a qualquer momento, que permite a colaboração entre os criadores de conteúdo.

Atualmente, a área de ferramentas de autoria conta com várias opções disponíveis para download (algumas gratuitas e outras pagas) e outras presentes apenas nas descrições de artigos científicos. A seção de trabalhos relacionados desse artigo descreveu algumas dessas ferramentas. Conforme apresentado, o UFC-Inventor se destaca dessas ferramentas por ser capaz de gerar aplicações com recursos de Computação Ubíqua para diferentes sistemas operacionais a partir de 
uma modelagem gráfica. Além disso, a abordagem se destaca por não demandar conhecimentos de programação, por utilizar uma especificação aberta e pelo fato das aplicações geradas não dependerem de conexão com Internet.

Além das ferramentas citadas na seção de trabalhos relacionados, outras iniciativas têm sido desenvolvidas no sentido de promover o uso dos sistemas de autoria na educação. A uAdventure é uma ferramenta voltada para a criação de jogos educacionais de aventura sem a necessidade de programação (Perez-Colado et al., 2017). Como diferenciais, a uAdventure conta com: utilização de técnicas de análise de aprendizagem (em inglês, learning analytics) para coleta e processamento dos dados dos jogos utilizados pelos alunos e disponibilização dos resultados para os professores; e, criação de jogos educativos móveis baseados em geolocalização, utilizando o GPS e a bússola do smartphone. Apesar de contar com recursos importantes, quando considerado o foco das aulas de campo, a uAdventure apresenta as seguintes limitações: não permite a utilização do sensor acelerômetro, para medição dos ângulos de mergulho das rochas; as aplicações geradas dependem de conexão com a Internet, o que muitas vezes não acontece em aulas de campo; e, não conta com recursos de compartilhamento das informações coletadas com outros colegas presentes na aula. Além disso, uma limitação relevante da uAdventure, que não está relacionada a aulas de campo, é o fato dela ser dependente de uma plataforma de terceiros que é paga: a Unity ${ }^{\circledR}$. Essa restrição pode limitar a capacidade de customização da ferramenta.

Em outro estudo, Ververidis et al. (2018) criaram uma ferramenta de autoria para possibilitar que educadores criassem laboratórios virtuais em três dimensões (3D). A construção dos laboratórios 3D se dá a partir da utilização dos templates contidos na ferramenta: química e energia eólica. Os laboratórios virtuais criados pela ferramenta são voltados para computadores pessoais e necessitam de conexão com a internet para funcionar. Essas características tornam proibitivo o uso dessa ferramenta em aulas de campo, que demandam a mobilidade proporcionada pelos dispositivos móveis e a independência da Internet, devido aos períodos frequentes de ausência de conexão.

Nesse sentido, o desenvolvimento do UFC-Inventor se destaca como umas das principais contribuições desse trabalho. Trata-se de uma ferramenta construída para professores que pretendem usar os recursos da computação ubíqua e dos dispositivos móveis dos alunos (como câmera, GPS, bússola e outros sensores) em suas aulas de campo, sem a obrigatoriedade de saber programar. Isso pôde ser verificado no estudo de usabilidade, que mostrou que todos os professores conseguiram gerar as aplicações propostas nas tarefas, mesmo apresentando diferenças de desempenhos entre os participantes (resposta à QP1). As opiniões dos professores indicaram que o sistema teve uma boa aceitação, com destaque ao interesse deles em continuar a usar o sistema. As observações apontadas (ausência de manual detalhado e impossibilidade de visualizar a interface do aplicativo em tempo de projeto) não inviabilizaram a utilização do UFCInventor e caracterizaram-se mais como sugestões de melhorias do que problemas propriamente ditos.

Outro resultado importante desse estudo foi o teste com alunos em campo utilizando uma aplicação projetada por um professor com o UFC-Inventor. Com esse teste, foi possível avaliar o funcionamento completo do sistema em uma situação real com estudantes. As respostas dos dezessete alunos aos instrumentos de avaliação demonstraram a capacidade do UFC-Inventor de gerar uma aplicação ubíqua, que foi aprovada pelos estudantes nos critérios de usabilidade e utilidade (resposta à QP2). 


\section{Conclusão}

Este artigo apresentou o sistema UFC-Inventor, uma solução para que professores consigam utilizar os recursos dos dispositivos móveis dos alunos e da Computação Ubíqua (como câmera, GPS, bússola e outros sensores) em suas aulas de campo. Isso foi verificado com as avaliações realizadas que demonstraram a modelagem e a geração automática de aplicações ubíquas multiplataforma sem a necessidade dos usuários terem conhecimentos específicos de programação. Os resultados dos testes realizados com professores e alunos, em condições reais de uso, demostraram o funcionamento completo do UFC-Inventor: do planejamento da aula a partir do modelo gráfico, passando pela geração da aplicação móvel, até utilização dela nos celulares dos alunos em campo. Além disso, os experimentos indicaram bons níveis de usabilidade e utilidade tanto pelo sistema quanto pela aplicação gerada.

Como trabalhos futuros, inicialmente pretende-se implementar as sugestões de melhorias apontadas nos testes pelos professores, como a impossibilidade de se visualizar a aplicação durante o planejamento na ferramenta e a ausência de um manual do usuário. Posteriormente, almeja-se realizar novos testes do UFC-Inventor com disciplinas de outras áreas (como Biologia, Ecologia e História), com o objetivo de se comprovar a aplicabilidade do sistema em outros contextos de aprendizagem.

\section{Referências}

Ainsworth, S., \& Grimshaw, S. (2004). Evaluating the REDEEM authoring tool: can teachers create effective learning environments?. International Journal of Artificial Intelligence in Education, 14(3, 4), 279-312. [GS Search]

Alvarez, V., Pérez-Pérez, J. R., Paule, M., \& de Freitas, S. (2016). Ariane: a web-based and mobile tool to guide the design of augmented reality learning activities. Mobile Learning FuturesSustaining Quality Research and Practice in Mobile Learning, 33. [GS Search]

Bangor, A., Kortum, P., \& Miller, J. (2009). Determining what individual SUS scores mean: Adding an adjective rating scale. Journal of usability studies, 4(3), 114-123. [GS Search]

Brambilla, M., Cabot, J., \& Wimmer, M. (2012). Model-driven software engineering in practice. Synthesis Lectures on Software Engineering, 1(1), 1-182. [GS Search]

Brooke, J. (1996). SUS-A quick and dirty usability scale. Usability evaluation in industry, 189(194), 4-7. [GS Search]

Chede, C. (2008). Padrões abertos interoperabilidade e interesse público. Revista Politics, 1(1). [GS Search]

Davis, F. D. (1985). A technology acceptance model for empirically testing new end-user information systems: Theory and results (Doctoral dissertation, Massachusetts Institute of Technology).[GS Search]

de Oliveira, A. P. L., \& Correia, M. D. (2013). Aula de campo como mecanismo facilitador do ensino-aprendizagem sobre os ecossistemas recifais em alagoas. Alexandria: Revista de Educação em Ciência e Tecnologia, 6(2), 163-190. [GS Search]

García, C. G., G-Bustelo, B. C. P., Espada, J. P., \& Cueva-Fernandez, G. (2014). Midgar: Generation of heterogeneous objects interconnecting applications. A Domain Specific 
Language proposal for Internet of Things scenarios. Computer Networks, 64, 143-158. [GS Search]

Garret, J. J. The Elements of User Experience: User-Centered Design for the Web and Beyond. Second Edition. Berkley: New Riders Press, 2011. [GS Search]

Giemza, A., Bollen, L., \& Hoppe, H. U. (2011). LEMONADE: field-trip authoring and classroom reporting for integrated mobile learning scenarios with intelligent agent support. International Journal of Mobile Learning and Organisation, 5(1), 96-114. [GS Search]

Gilman, E., Milara, I. S., Cortés, M., \& Riekki, J. (2015). Towards user support in ubiquitous learning systems. IEEE Transactions on Learning Technologies, 8(1), 55-68. [GS Search]

Gómez, S., Zervas, P., Sampson, D. G., \& Fabregat, R. (2014). Context-aware adaptive and personalized mobile learning delivery supported by UoLmP. Journal of King Saud University-Computer and Information Sciences, 26(1), 47-61. [GS Search]

Hwang, G. J., \& Wong, L. H. (2014). Guest Editorial: Powering Up: Insights from Distinguished Mobile and Ubiquitous Learning Projects across the World. Educational Technology \& Society, 17(2), 1-3. [GS Search]

Koper, R., \& Olivier, B. (2004). Representing the learning design of units of learning. Journal of Educational Technology \& Society, 7(3). [GS Search]

Marçal, E., Viana, W., Andrade, R. M., \& Rodrigues, D. (2014, October). A mobile learning system to enhance field trips in geology. In Frontiers in Education Conference (FIE), 2014 IEEE (pp. 1-8). IEEE. [GS Search]

Marçal, E., Andrade, R., \& Viana, W. (2015, October). ML4UL: Uma Extensão ao Padrão IMS LD para Modelagem de Sistemas Móveis e Ubíquos para Aulas de Campo. In Brazilian Symposium on Computers in Education (Simpósio Brasileiro de Informática na EducaçãoSBIE) (Vol. 26, No. 1, p. 160). [GS Search]

Perdikuri, K. (2014, October). Students' Experiences from the use of MIT App Inventor in classroom. In Proceedings of the 18th Panhellenic Conference on Informatics (pp. 1-6). ACM. [GS Search]

Perez-Colado, I. J., Perez-Colado, V. M., Martínez-Ortiz, I., Freire-Moran, M., \& FernándezManjón, B. (2017, April). UAdventure: The eAdventure reboot: Combining the experience of commercial gaming tools and tailored educational tools. In Global Engineering Education Conference (EDUCON), 2017 IEEE (pp. 1755-1762). IEEE. [GS Search]

Rachid, C. L., \& Ishitani, L. (2012). M-tutorial: ferramenta de autoria para desenvolvimento de tutoriais voltados para o m-learning. Revista Brasileira de Informática na Educação (RBIE), 20(1). [GS Search]

Rocha, L et al., Ubiquitous Software Engineering: Achievements, Challenges and Beyond, 2011 25th Brazilian Symposium on Software Engineering, Sao Paulo, 2011, pp. 132-137. [GS Search]

Roslin, M. A. Y. A., Maga, J. P., Rosales, A. B., Cereno, R., \& Tapay, N. E. (2009). Social impact of ecotourism on the behavior of students on educational field trips to Makiling Botanic Gardens in the University of the Philippines Los Baños. USM R\&D Journal, 17(1), 71-80. [GS Search] 
Shakil, A. F., \& Hafeez, S. (2011). The need and importance of field trips at higher level in Karachi, Pakistan. International Journal of Academic Research in Business and Social Sciences, 2(1), 1. [GS Search]

Sharples, M. Taylor, J., Vavoula, G.. A Theory of Learning for the Mobile Age. R. Andrews and C. Haythornthwaite. The Sage Handbook of Elearning Research, Sage publications, pp.221247, 2006. [GS Search]

Singh, T. K. (2018). Importance Of Authoring Tools In Digital World. Magic EdTech. Disponível em https://www.magicedtech.com/blog/importance-of-authoring-tools-in-digital-world/

Tabuenca, B., Kalz, M., Ternier, S., \& Specht, M. (2016). Mobile authoring of open educational resources for authentic learning scenarios. Universal Access in the Information Society, 15(3), 329-343. [GS Search]

Ververidis, D., Chantas, G., Migkotzidis, P., Anastasovitis, E., Papazoglou-Chalikias, A., Nikolaidis, E., \& Liapis, A. (2018). An authoring tool for educators to make virtual labs. In Proc. Internat. Conf. on Interactive Collaborative Learning. [GS Search]

Viana, W., Andrade, R. M. C., Maia, M., Marçal, E., Gensel, J., Lima, J. V., \& Sanchez, J. (2011). Towards context-aware and mobile e-learning application. In TISE 2011, Congreso Internacional de Informática Educativa. [GS Search]

Vygotsky, L. S. (1978). Mind in society: the development of higher psychological processes.Cambridge: Harvard University Press. [GS Search]

Weiser, M. (1999). The computer for the 21st century. SIGMOBILE Mob. Comput. Commun. Rev. 3, 3 (July 1999), 3-11. [GS Search]

Wu, P. H., Hwang, G. J., \& Tsai, W. H. (2013). An expert system-based context-aware ubiquitous learning approach for conducting science learning activities. Journal of Educational Technology \& Society, 16(4). [GS Search]

Xie, B., \& Abelson, H. (2016, September). Skill progression in MIT app inventor. In IEEE Symposium on Visual Languages and Human-Centric Computing (VL/HCC), 2016 (pp. 213-217). IEEE. [GS Search]

Zbick, J., Nake, I., Jansen, M., \& Milrad, M. (2014, November). mLearn4web: a web-based framework to design and deploy cross-platform mobile applications. In Proceedings of the 13th International Conference on Mobile and Ubiquitous Multimedia (pp. 252-255). ACM. [GS Search] 